\title{
THE OSCILLATIONS AND THE STABILITY OF ROTATING MASSES WITH MAGNETIC FIELDS
}

\author{
R. K. KOCHHAR and S. K. TREHAN
}

Dept. of Mathematics, Panjab Universlty, Chandigarh, India

\begin{abstract}
It is shown that when a magnetic field is present along the axis of rotation, the point of bifurcation, where the Jacobi ellipsoids branch off from the Maclaurin spheroids, occurs at a value of eccentricity higher than the value $e=0.81267$ that obtains in the absence of a magnetic field. This is in contrast with the effect of a toroidal magnetic field which, as has been shown earlier, leaves the point of bifurcation unaffected.
\end{abstract}

